\title{
Modeling compositional dynamics based on GC and purine contents of protein-coding sequences
}

Zhang Zhang, Jun Yu*

\begin{abstract}
Background: Understanding the compositional dynamics of genomes and their coding sequences is of great significance in gaining clues into molecular evolution and a large number of publically-available genome sequences have allowed us to quantitatively predict deviations of empirical data from their theoretical counterparts. However, the quantification of theoretical compositional variations for a wide diversity of genomes remains a major challenge.
\end{abstract}

Results: To model the compositional dynamics of protein-coding sequences, we propose two simple models that take into account both mutation and selection effects, which act differently at the three codon positions, and use both GC and purine contents as compositional parameters. The two models concern the theoretical composition of nucleotides, codons, and amino acids, with no prerequisite of homologous sequences or their alignments. We evaluated the two models by quantifying theoretical compositions of a large collection of protein-coding sequences (including 46 of Archaea, 686 of Bacteria, and 826 of Eukarya), yielding consistent theoretical compositions across all the collected sequences.

Conclusions: We show that the compositions of nucleotides, codons, and amino acids are largely determined by both GC and purine contents and suggest that deviations of the observed from the expected compositions may reflect compositional signatures that arise from a complex interplay between mutation and selection via DNA replication and repair mechanisms.

Reviewers: This article was reviewed by Zhaolei Zhang (nominated by Mark Gerstein), Guruprasad Ananda (nominated by Kateryna Makova), and Daniel Haft.

\section{Background}

Compositional biases in the contexts of nucleotides, codons, and amino acids are found among bacteria [1-4], fungi [5,6], insects [7-10], plants [11,12], and vertebrates $[13,14]$, which presumably arise from unbalanced forces of mutation and selection and are maintained by the species in their populations [15-17]. For any individual gene, its compositional biases reflect the action of both mutation and selection, which is also linked to the abundance of iso-accepting transfer RNAs and the catalytic efficiencies of their synthetases, thereby translation efficiencies [2,6,18-22]. Therefore, composition analysis is of great significance in better

\footnotetext{
* Correspondence: Jun.Yu@Kaust.edu.sa

Plant Stress Genomics Research Center, Division of Chemical and Life Sciences and Engineering, King Abdullah University of Science and Technology, Thuwal 23955-6900, Kingdom of Saudi Arabia
}

understanding compositional dynamics in order to provide evidence for molecular evolution [23,24].

Nucleotide compositions are highly variable among genomes, and the guanine-plus-cytosine ( $\mathrm{G}+\mathrm{C}$ or simply GC) content differs dramatically from one species to another [25-27], particularly among bacterial genomes, which varies from $17 \%$ (Candidatus Carsonella ruddii $\mathrm{PV}$ ) to $75 \%$ (Anaeromyxobacter dehalogenans 2CP-C) $[28,29]$. The dynamics of nucleotide compositions is also coupled closely to codon and amino acid compositions. The most likely factor that determines codon/amino acid usage is mutational bias that shapes GC composition constantly when genomes are either replicated or repaired by DNA polymerases [30]. In addition, empirical relationships between GC content and codon/amino acid usage have been documented in many species [25,31-38], providing ample data for theoretical modeling and simulation studies. Despite an attempt to model 
compositional dynamics from GC content alone [31], there has been little interpretations for the effect of mutation and selection at different codon positions as well as the contribution of purine content. Based on the Chargaff's rules $[39,40]$ that the amount of adenine equals to thymine and the amount of guanine equals to cytosine (viz., $A=T$ and $G=C$ ), purine content is expected to be centered narrowly around $50 \%$ since $\mathrm{A}+$ $\mathrm{T}+\mathrm{G}+\mathrm{C}=2(\mathrm{~A}+\mathrm{G})=100 \%$, in contrast to a broad variation of GC content. Although purine content is thought to be nearly constant, deviating in minimal ways toward both low and high around $50 \%$, its subtle yet fundamental variation can lead to a considerable departure from the base-pairing rule of $A=T$ and $G=$ $\mathrm{C}$ and consequently provoke differential codon and amino acid usages.

Here we present two models that calculate theoretical compositions of nucleotides, codons, and amino acids in quantitative ways. Our models assume that mutation and selection act at the level of nucleotide (rather than codon or amino acid) [41], take into account of diverse forces from both mutation and selection at three codon positions, and employ GC and purine contents as two essential parameters to model compositional dynamics and quantify theoretical compositions without the requisite of orthologous sequences or their alignments. We examine the effectiveness of our models on a large collection of protein-coding sequences across the three domains of life (including 46 of Archaea, 686 of Bacteria, and 826 of Eukarya) and provide an in-depth discussion on the theoretical composition dynamics through comparisons of the observed compositions to the empirical data.

\section{Results}

We obtained a large collection of codon usage data across the three domains of life (46 archaea, 686 bacteria, and 826 eukaryotes). Both models (Model 1 and Model 2; see Figure 1) use GC (S) and purine (R) contents to predict expected compositions theoretically. Different from Model 2, Model 1 requires prior knowledge of empirical relationships between $\mathrm{S}$ and $\mathrm{S}_{i}$ and between $\mathrm{R}$ and $\mathrm{R}_{i}$, where $i$ represents codon position $(i=1,2,3)$ (see Models). We inferred these empirical relationships (Additional file 1) from all the collected sequences in individual domains of life for Model 1.

\section{Nucleotide composition}

We plotted the expected and observed frequencies of the four nucleotides and their individual frequencies at the three codon positions against GC content for all data in our collection (Additional file 2). An example for guanine is shown in Figure 2. Both models performed well across a wide range of $\mathrm{GC}$ contents and yielded very close predictions for the nucleotide $\mathrm{G}$ (Figure $2 \mathrm{~A}$ to $2 \mathrm{C}$ ) and for the three codon positions (Figure 2D to $2 \mathrm{~L}$ ). The expected compositions with changing GC contents exhibit similar trends as compared to the observed ones, despite the fact that deviations at the second codon position appeared more pronounced in comparison with the first and third codon positions (Figure 2G to 2I; discussed below). Furthermore, the expected compositions predicted by Model 1 correlated with GC content linearly, whereas those predicted by Model 2 appeared scattered around those by Model 1, indicating greater deviations. Taken together, the two models produced close predictions for the expected nucleotide compositions, exhibiting comparable trends with the observed (Figure 2 and Additional file 2).

\section{Codon composition}

We further used the models to predict the codon compositions (see Models). The expected and observed codon frequencies were plotted against GC content over all collected sequences (Additional file 3). We took four randomly selected codons (AAT, TGC, GCC, and CTT) as examples (Figure 3). When GC content varies from low to high, both models show consistent predictions for expected codon compositions that are very similar to the distributions of the observed (Figure 3A to 3L). Specifically, the expected compositions of codons AAT and CTT yield negative correlations with the increasing GC content, agreeing well with the observed (Figure $3 \mathrm{~A}$ to $3 \mathrm{C}$ and $3 \mathrm{~J}$ to $3 \mathrm{~L}$ ). In contrast, the expected compositions of TGC and GCC codons correlate positively with the increasing GC content, again consistent with the observed (Figure 3D to 3F and 3G to 3I). Moreover, in comparison with Model 2, the predicted trends by Model 1 are smoother when the GC content varies (Figure 3). Although there are deviations between the expected and observed in general, the two models predict rather consistent codon compositions (Figure 3 and Additional file 3).

\section{Amino acid composition}

Based on the expected codon compositions, we compared the expected and observed amino acid compositions across the three domains of life (Additional file 4). We chose to show here the plots for codons AAT, TGC, GCC and CTT for four amino acids, Asn (asparagine), Cys (cysteine), Ala (alanine), and Leu (leucine), respectively (Figure 4). Although predicting amino acid compositions may be entangled by the fact that most amino acids are encoded by multiple codons and thus may involve greater deviations, both models still performed moderately well in quantifying the expected amino acid compositions. The expected compositions of Asn (encoded by AAT and AAC) decreased with increasing GC content, providing comparable trends 


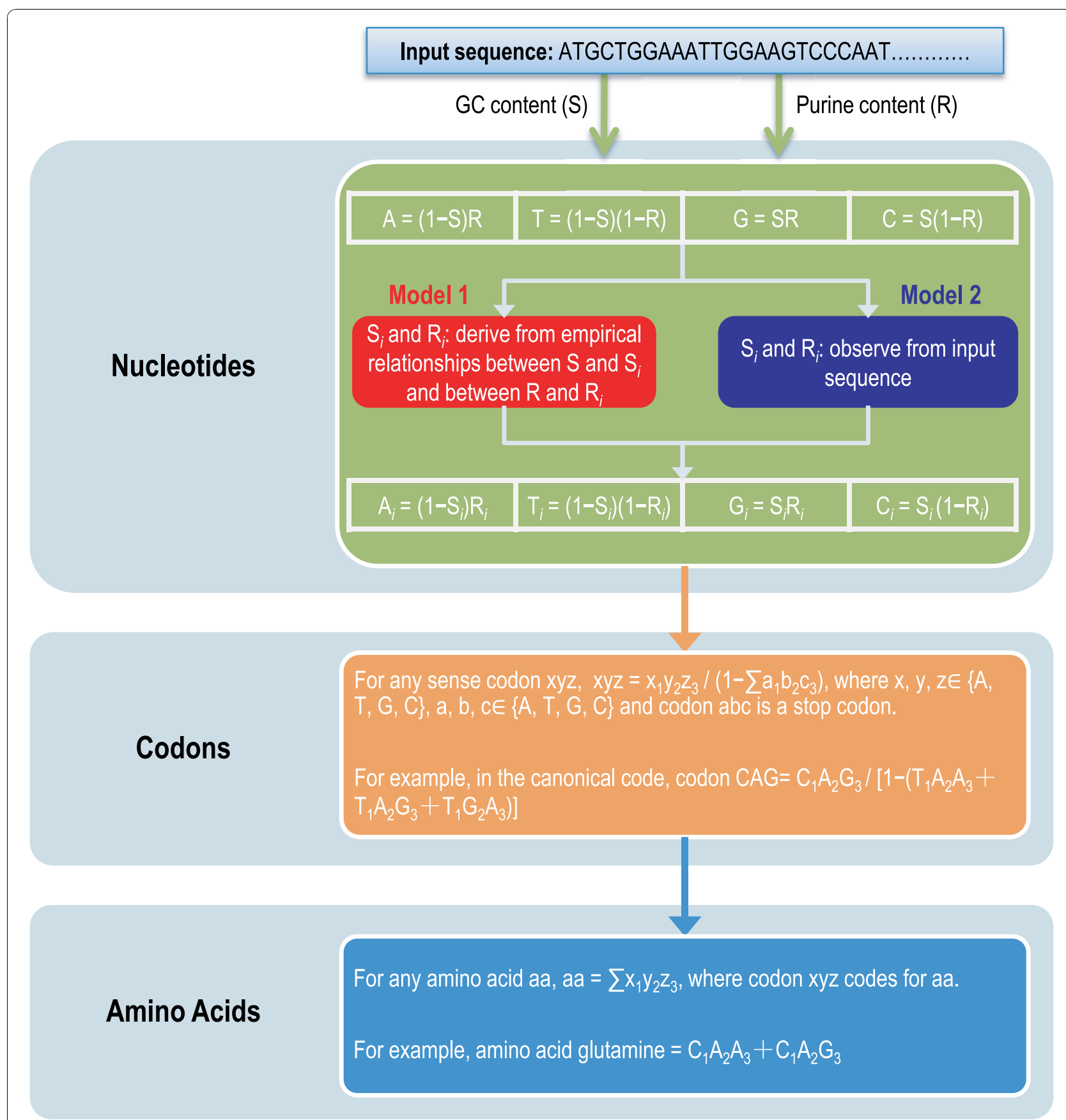

Figure 1 Illustrations for quantifying theoretical compositions. Quantification of theoretical compositions of nucleotide, codon, and amino acid is based on GC (S) and purine (R) contents, which are readily observed from input coding sequences. Model 1 (red) and Model 2 (blue) differ only in how position-dependent $G C\left(S_{i}\right)$ and purine $\left(R_{i}\right)$ contents are calculated, where $i$ represents codon position $(i=1,2,3)$.

with the observed (Figure 4A to $4 \mathrm{C}$ ). In contrast, the expected compositions of Cys (encoded by TGT and TGC) appeared constant (extremely low) with changing GC content, displaying similar trends with the observed (Figure 4D to 4F), albeit slightly larger than the observed. As Ala is encoded by codons GCN (where $\mathrm{N}=\mathrm{A}, \mathrm{T}, \mathrm{G}, \mathrm{C})$, the expected compositions of Ala dramatically increased with increasing GC content, but appeared smaller than the observed (especially in bacteria; discussed below); nevertheless, the expected compositions of Ala still presented similar trends with the observed (Figure 4G to 4I). With regard to Leu (encoded by six different codons, CTN and TTR), its observed compositions appear much more scattered 


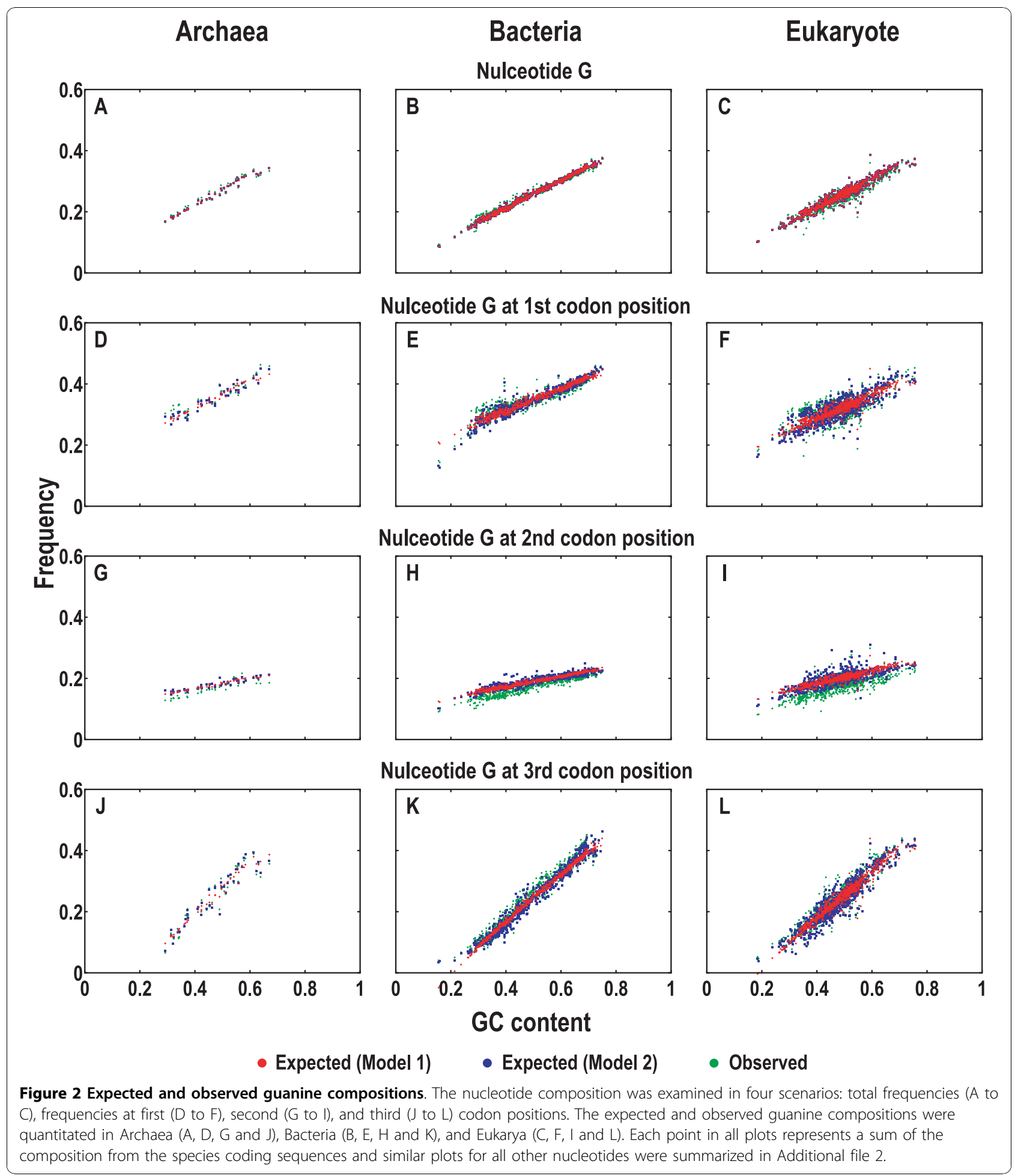

than those of Asn, Cys or Ala. Even so, both models are still capable of predicting consistent compositions for Leu. Although the expected compositions of Leu are smaller than the observed in archaea and bacteria, they appear closer to the observed in eukaryote (Figure $4 \mathrm{~J}$ to $4 \mathrm{~L})$. Additionally, comparing the expected amino acid compositions between the two models, we found that Model 1 again exhibits smoother trends (except Leu) tailored to the increasing GC content. Collectively, the two models also offered a consistent quantification for amino acid compositions across the three domains of life (Figure 4 and Additional file 4). 


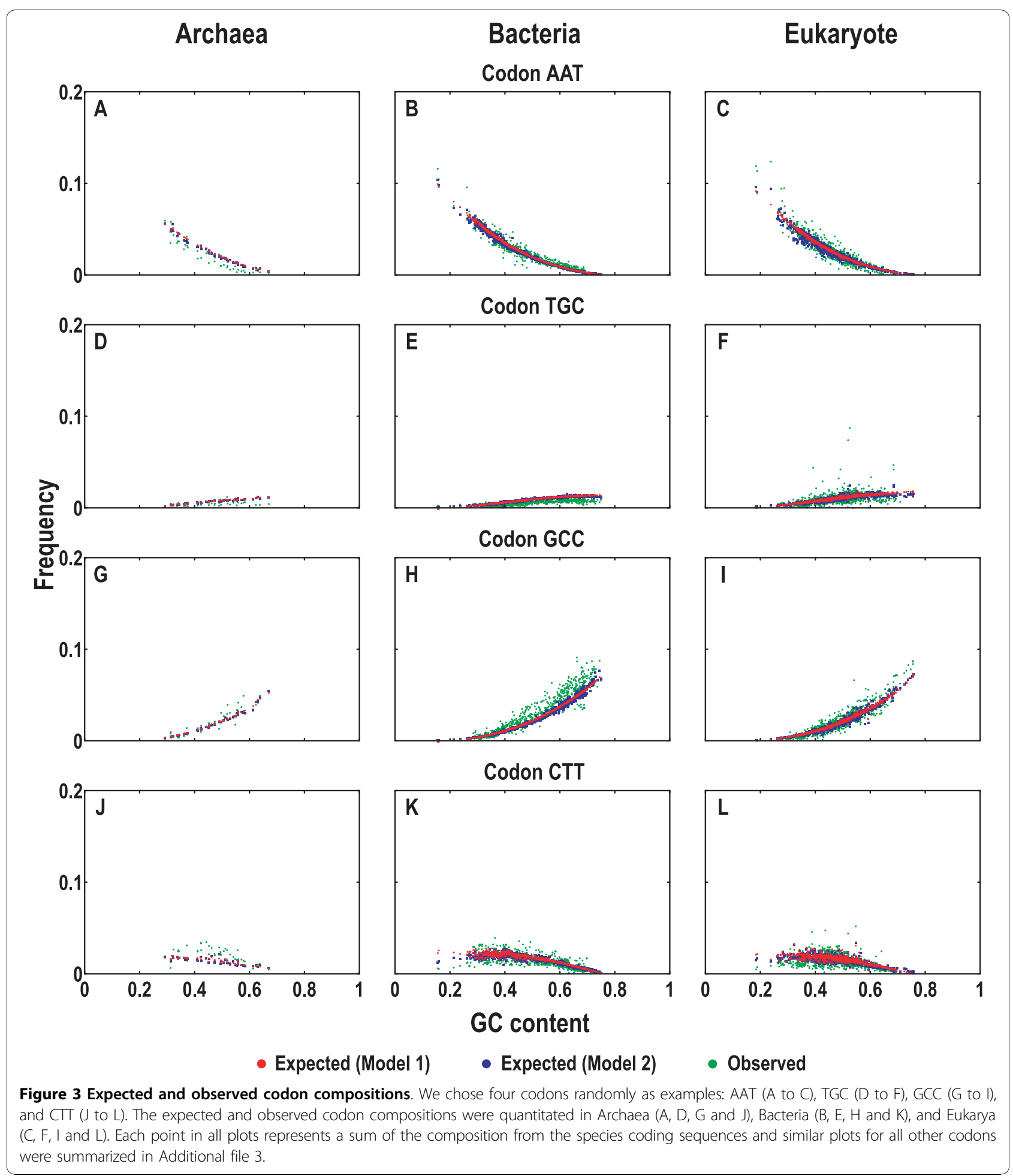

\section{Discussion}

\section{Significances of incorporating GC and purine contents} into models

Empirical relationships between GC content and codon (amino acid) usage have been widely reported but explained in most of the cases less comprehensively.
Here we show that each codon as well as each nucleotide in cellular genomes follows a very similar trend when GC content varies (Figures 2, 3, 4 and Additional files 2, 3, 4), albeit lesser differences between prokaryotes and eukaryotes due to their sequence heterogeneity (for example, isochores in vertebrates [42,43], 


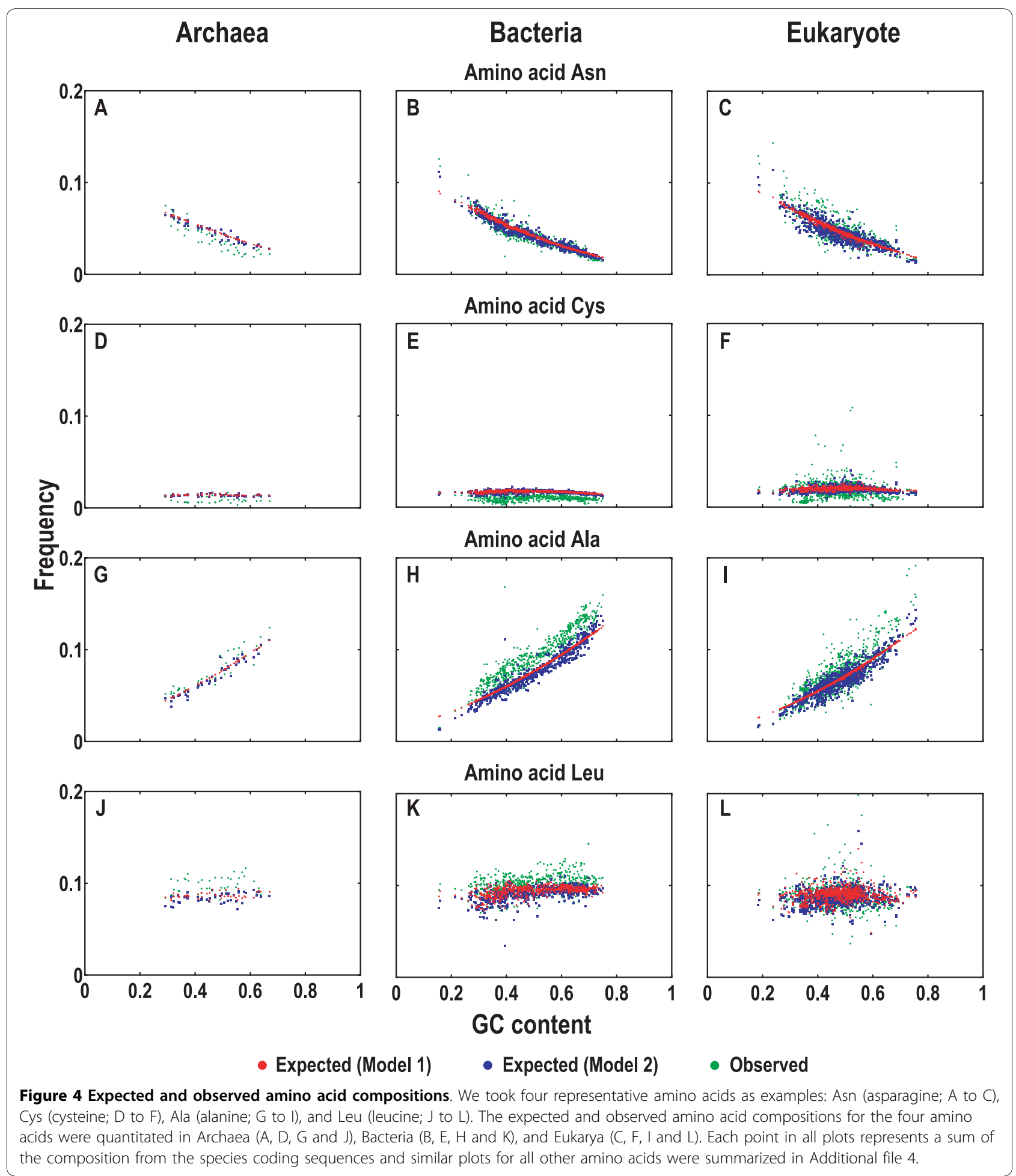

integral membrane proteins with hydrophobic nature, horizontal transfer of DNA and questionable predicted coding regions, etc.). Our results strongly suggest that mutation and selection not only act at different levels but also exhibit different priorities that are attributable to the organization of the genetic code $[44,45]$. At the nucleotide level, we observe that the compositions of all species for a given GC content are very similar and more or less predictable. Consequently, GC content becomes a significant predictor for nucleotide, codon, and amino acid compositions, since half of the amino acids are rather GC content-sensitive in their first and 
second codon positions [44-47]. However, it does not mean that GC content, varying from $17 \%$ to $75 \%$, is the sole determinant of compositions at all levels [31,48]; purines have been widely reported to have a determinative role in amino acid physicochemical properties and purines in the second codon position may control the charge and hydrophobicity of amino acids [44,46,49,50]. Similar to GC content, purine content also differs from one species to another, albeit with a relatively smaller range in a nearly $10 \%$ deviation below or above the half line. In bacteria, for instance, the minimum of purine content is $48.0 \%$ for Clavibacter michiganensis subsp. michiganensis NCPPB 382, whereas the maximum is $58.8 \%$ for Clostridium tetani E88. The slight deviation of purine content, indicating a complex interplay of mutation and selection and reflecting an important balance between the purine-sensitive and insensitive amino acids -15 and 5 (as signified by their codons' sensitivity to purine variations at the third codon position), respectively [51]-can give rise to completely different compositions at the levels of both codons and amino acids (as indicated in Equations 1-8).

Therefore, our models first adopt GC and purine contents as two important compositional elements and consider heterogeneous mutation and selection forces acting at all three codon positions. As testified across a wide variety of species, the models provided consistent compositions, quantitatively recapturing the empirical relationships with changing GC and purine contents. Our results, especially in the various changing trends (most of them are not linear) further validated that mutations (dominated by GC content variations) and selections (dominated by purine content variations) mainly act at the level of nucleotides rather than codons or amino acids in accordance with previous studies $[12,41,52]$. Although our models are designed to work on protein coding sequences, it might also be applicable to nucleotide frequencies in non-coding sequences as an alternative. Second, the deviations from the dominant trends for certain amino acids, to a lesser extent some of their codons (such as it is well-accepted that purine-rich sequences often serve as elements of exonic enhancers among animal genes that have multiple spliceosomal introns), reflect selection forces acting primarily on certain amino acids of the proteomes when their amino acid sequence changes interfere with protein level functions. Third, there are other balancing forces buried in the organization of the genetic code. One of the sets includes the six-fold codons for Leu, Arg (arginine), and Ser (serine). All of them provide diverse balances for purine content variations as they are all divided between the purine-sensitive and insensitive codons [44,46]. Although four of the codons for Arg are in the GC-rich quarter of the genetic code, its counterpart, Lys (lysine) has all its codons in the $\mathrm{AT}$-rich quarter in order to maintain enough basic amino acids in the proteomes [44].

Our models have several variants. Since they are built on the basis of GC and purine contents and thus symbolized as $\{\mathrm{GC}, \mathrm{AG}\}$ or $\{\mathrm{S}, \mathrm{R}\}$, their variants can also be represented by $S$ and $R:\{A T, A G\}=\left\{S^{\mathrm{c}}, R\right\},\{G C, T C\}=$ $\left\{S, R^{c}\right\},\{A T, T C\}=\left\{S^{c}, R^{c}\right\}$. As assumed, $S$ and $R$ is an independent pair, which leads to $S^{c}$ and $R, S$ and $R^{c}, S^{c}$ and $R^{c}$ are also independent pairs (see Models). Therefore, the variants, $\left\{S^{c}, R\right\},\left\{S, R^{c}\right\},\left\{S^{c}, R^{c}\right\}$, are in essence equivalent to our models.

\section{Implications of composition deviations}

The expected compositions predicted by our models, however, sometimes deviate in various degrees from the observed. Such deviations can be caused by complex evolutionary mechanisms (e.g., extreme dinucleotide abundance [53]) and deciphered in terms of mutation and selection [54,55]; mutation towards a particular nucleotide content (e.g., GC content) primarily determines codon and amino acid usage according to the genetic code structure [56] and selection essentially caters for a given amino acid usage [57]. Therefore, it is likely that these composition deviations provide implications for molecular evolution.

Considering nucleotide compositions at all three codons positions (Figure 2 and Additional file 2), four nucleotides at the first and third codon positions deviated evenly, suggesting stronger mutation effects. On the contrary, four nucleotides at the second codon position deviated remarkably, exhibiting a similar manner in all species. As compared to the expected compositions, $\mathrm{A}$ and $\mathrm{C}$ appear overestimated, whereas $\mathrm{G}$ and $\mathrm{T}$ are underestimated (Figure 2 and Additional file 2). This indicates the strong selection acting at the second position that is intrinsic to the organization of the genetic code; amino acids that have $\mathrm{A}$ or $\mathrm{C}$ at their second codon positions are more diverged and less flexible toward nucleotide changes across codon positions than within codon positions. Conversely, the amino acids that have $\mathrm{G}$ or $\mathrm{T}$ at their second codon positions are relatively relaxed toward nucleotide changes across codon positions. Most noticeable are Leu and Arg, whose codons are partitioned within the same position but between the purine-sensitive and insensitive halves (Additional file 5) [44]. Our results are in agreement with previous observations $[41,44,58]$.

Since selection forces largely act at the levels of amino acids and their codons, we are able to assess the degrees of selection in different organisms by calculating subtle differences among amino acid (codons) conversion matrices. For instance, Ala and Val (valine) are the two most departed amino acids in all the collected 
sequences. Namely, in comparison to expectations, there are a surplus of alanine and a deficit of valine. Since amino acids are exchanged at different frequencies due to their compositional relevance at nucleotide level, it is possible that deviations of these two amino acids are highly related to such exchangeability. Therefore, we constructed five amino acid exchange matrices that are based on five different datasets in Escherichia coli, fruit fly, rice, yeast, and mammal (see Methods). When we take the top 10 highly-exchangeable pairs in all five matrices, the four among the top are (1) Ala $\leftrightarrow$ Ser, (2) $\mathrm{Ala} \leftrightarrow \mathrm{Thr}$ (threonine), (3) Ala $\leftrightarrow \mathrm{Val}$, and (4) Val $\leftrightarrow$ Ile (isoleucine) (Additional file 6). As we know, amino acids with similar physicochemical properties tend to be more exchangeable [59-62]. It appears that Ala is the most active amino acid, primarily due to the fact that several of its neighboring amino acids have similar physicochemical properties (such as their size parameters). With regard to the exchange between Val and Ile, it is their similarity in hydrophobicity that plays a key role. These results are by and large consistent with findings in several previous studies $[12,63,64]$. Therefore, our models bear significance in establishing a theoretical framework for compositional analysis and providing clues for molecular evolution studies.

\section{Conclusions}

Here we have presented two models that theoretically quantify expected compositions of nucleotides, codons, and amino acids, based merely on GC and purine contents (which are easily computed from input sequences). We evaluated the two models on a large collection of protein-coding sequences across the three domains of life. Our results show that the two models are capable of yielding consistent expected compositions. In addition, our results indicate that deviations of the observed from the expected compositions are signatures resulted from complex interplays between mutation and selection. Therefore, our models represent a promising theoretical framework for compositional studies.

\section{Methods}

Models

We devise two models (denoted as Model 1 and Model 2) that theoretically quantify expected compositions of nucleotides, codons, and amino acids, beginning with only GC and purine contents, which are easily derived from input sequences. To provide a better description, we detailed the computational procedures in Figure 1, and since we only use the coding sequence for all the analyses, any nucleotide content (GC or purine content) used is referred to coding sequences.

To describe the two models, let us briefly recapitulate several pertinent elements in probability theory. Suppose that the universal set is $\mathrm{U}$, and $\mathrm{X}$ or $\mathrm{Y}$ are two subsets of $U$, there are three common operations: (1) union of $\mathrm{X}$ and $\mathrm{Y}$, denoted $\mathrm{X} \cup \mathrm{Y}$, is the set of all elements that are a member of $\mathrm{X}$, or $\mathrm{Y}$, or both, viz., $\mathrm{X} \cup \mathrm{Y}=\{z \mid z \in \mathrm{X}$ or $z \in \mathrm{Y}\}$; (2) intersection of $\mathrm{X}$ and $\mathrm{Y}$, denoted $\mathrm{X} \cap \mathrm{Y}$, is the set of all elements that are members of both $X$ and $\mathrm{Y}$, viz., $\mathrm{X} \cap \mathrm{Y}=\{z \mid z \in \mathrm{X}$ and $z \in \mathrm{Y}\}$; (3) complement of set $X$ relative to set $U$, denoted $X^{c}$, is the set of all members of $\mathrm{U}$ that are not members of $\mathrm{X}$, viz., $\mathrm{X}^{\mathrm{c}}=\{z \mid z$ $\notin X\}$. For simplicity, the four nucleotide (adenine, thymine, guanine, and cytosine) contents are denoted as $\mathrm{A}$, T, G, and C, respectively. Since our models are built at the nucleotide level, the universal set $\mathrm{U}$ is defined as $\mathrm{U}$ $=\{\mathrm{A}, \mathrm{T}, \mathrm{G}, \mathrm{C}\} . \mathrm{GC}$ and purine contents are denoted as $\mathrm{S}$ and $\mathrm{R}$, respectively, where $\mathrm{S}=\mathrm{G} \cup \mathrm{C}$ and $\mathrm{R}=\mathrm{A} \cup \mathrm{G}$. Notations used to describe in our models are summarized in Table 1.

In this study, $\mathrm{S}$ and $\mathrm{R}$ are assumed statistically independent. Statistical independence of two variables probabilistically means that the occurrence (value) of one variable does not change the probability for that (value) of the other. According to the Chargaff's rules again, $\mathrm{S}$ varies broadly whereas $R$ always centers at $50 \%$, implying the independence between $\mathrm{S}$ and $\mathrm{R}$. As $\mathrm{S}$ and $\mathrm{R}$ are statistically independent (that is, $\mathrm{S}$ and $\mathrm{R}$ form an independent pair), $\mathrm{S} \cap \mathrm{R}$ can be quantitatively expressed by $\mathrm{SR}$, namely, $\mathrm{S} \cap \mathrm{R}=\mathrm{SR}$, which is also applicable to the three pairs: $S^{c}$ and $R, S$ and $R^{c}$, and $S^{c}$ and $R^{c}$ [65]. As a result, each nucleotide is formulated as a function of $S$ and $\mathrm{R}$ (Equations 1-4). As $\mathrm{S}$ and $\mathrm{R}$ are to be calculated from input sequences, compositions of four nucleotides are readily inferred according to Equations 1-4. Obviously, a special case when $\mathrm{R}=0.5$, can lead to $\mathrm{A}=$ $\mathrm{T}=(1-\mathrm{S}) / 2, \mathrm{G}=\mathrm{C}=\mathrm{S} / 2$, which is consistent with the Chargaff's rule $[39,40]$.

\section{Table 1 Notation}

\begin{tabular}{ll}
\hline Parameter & Description \\
\hline $\mathrm{A}$ & Adenine content \\
$\mathrm{T}$ & Thymine content \\
$\mathrm{G}$ & Guanine content \\
$\mathrm{C}$ & Cytosine content \\
$\mathrm{GC}$ & Guanine-plus-cytosine content, $\mathrm{GC}=\mathrm{G}+\mathrm{C}$ \\
$\mathrm{S}$ & Guanine-plus-cytosine content, $\mathrm{S}=\mathrm{G}+\mathrm{C}$ \\
$\mathrm{R}$ & Purine (adenine-plus-guanine) content, $\mathrm{R}=\mathrm{A}+\mathrm{G}$ \\
$\mathrm{A}_{i}$ & Adenine content at codon position $i, i=1,2,3$ \\
$\mathrm{~T}_{i}$ & Thymine content at codon position $i, i=1,2,3$ \\
$\mathrm{G}_{i}$ & Guanine content at codon position $i, i=1,2,3$ \\
$\mathrm{C}_{i}$ & Cytosine content at codon position $i, i=1,2,3$ \\
$\mathrm{~S}_{i}$ & Guanine-plus-cytosine at codon position $i, \mathrm{~S}_{i}=\mathrm{G}_{i}+\mathrm{C}_{i}$ \\
& $i=1,2,3$ \\
$\mathrm{R}_{i}$ & Purine content at codon position $i, \mathrm{R}_{i}=\mathrm{A}_{i}+\mathrm{G}_{i,} i=1,2,3$ \\
\hline
\end{tabular}




$$
\begin{aligned}
& A=(A \cup T) \cap(A \cup G)=(G \cup C)^{c} \cap(A \cup G)=S^{c} \cap R=(1-S) R(1) \\
& T=(A \cup T) \cap(T \cup C)=(G \cup C)^{c} \cap(A \cup G)^{c}=S^{c} \cap R^{c}=(1-S)(1-R) \\
& G=(G \cup C) \cap(A \cup G)=S \cap R=S R \\
& C=(G \cup C) \cap(T \cup C)=(G \cup C) \cap(A \cup G)^{c}=S \cap R^{c}=S(1-R)
\end{aligned}
$$

Mutation and selection forces are assumed to act at nucleotide level, which also alter codon and amino acid usages [31,41]. Considering differential effects of mutation and selection at the three different codon positions [66], both models necessitate $S$ and $R$ at three codon positions, that is, $\mathrm{S}_{i}$ and $\mathrm{R}_{i}$, where $i$ is the codon position, $i=1,2$, 3. The two models proposed in this study differ only in how to compute $S_{i}$ and $\mathrm{R}_{i}$. As $\mathrm{S}$ and $\mathrm{R}$ are calculated from each individual sequence, Model 1 deduces $S_{i}$ and $\mathrm{R}_{i}$ from empirical relationships between $\mathrm{S}$ and $\mathrm{S}_{i}$ and between $\mathrm{R}$ and $\mathrm{R}_{i}$, where $\mathrm{S}_{i}$ and $\mathrm{R}_{i}$ are based on the linear relationships derived from multiple species $[31,32,35,37,67,68]$. Contrastingly, Model 2 simply counts $S_{i}$ and $R_{i}$ from the input sequence, in a manner similar to $S$ and R. After $S_{i}$ and $R_{i}$ being obtained, nucleotide contents at the three codon positions are inferred in the same way,

$$
\begin{aligned}
& \mathrm{A} i=\left(1-\mathrm{S}_{i}\right) \mathrm{R} i \\
& \mathrm{~T} i=\left(1-\mathrm{S}_{i}\right)(1-\mathrm{R} i), \\
& \mathrm{G} i=\mathrm{S}_{i} \mathrm{R}, \\
& \mathrm{C}_{i}=\mathrm{S}_{i}(1-\mathrm{R} i),
\end{aligned}
$$

where $\mathrm{A}_{i}, \mathrm{~T}_{i}, \mathrm{G}_{i}$, and $\mathrm{C}_{i}$ represent their corresponding contents at the three codon positions $(i=1,2,3)$.

Therefore, for any sense codon $x y z, x, y, z \in\{A, T, G$, $C$, its expected composition is given by the product of its constituent nucleotide frequencies $x_{1} y_{2} z_{3}$, normalized by the sum over all non-stop codons, $\mathrm{x}_{1} \mathrm{y} 2 \mathrm{z} 3 /\left(1-\sum_{\text {abc is a stop codon }} \mathrm{a}_{1 \mathrm{~b} 2 \mathrm{C} 3}\right)$, where $\mathrm{x}_{1}, \mathrm{y}_{2}$ and $\mathrm{z}_{3}$ are calculated from Equations 5-8. Amino acid composition relates closely to codon composition; for a given amino acid, its expected composition is the sum over all its codons.

Model 1, as it incorporates empirical relationships, is a combination of empirical and mechanistic, whereas Model 2 is purely mechanistic without any prior knowledge (Figure 1; see [69] for a review on models). As expected, therefore, Model 1 yields smoother compositions than Model 2 (see below). Model 1 is thus regarded as a specialized case of Model 2; without the prior knowledge, Model 2 has to be used in predicting theoretical compositions.

\section{Data Collection}

We retrieved species and their corresponding codon usage totals from Codon Usage Database (http://www. kazusa.or.jp/codon/) [70], which was tabulated from NCBI GenBank Flat File Release 160.0. We excluded species with less than 64 coding sequences tabulated from nuclear DNA, in order to ensure a sufficient sample size for estimating compositions of nucleotide, codon and amino acid. Species with alternative genetic codes were also eliminated from this study. As a consequence, we obtained a collection of 46 archaea, 686 bacteria, and 826 eukaryotes tabulated in Codon Usage Database. Composition frequencies were computed by exclusion of stop codons.

\section{Construction of amino acid exchange matrix}

To construct amino acid exchange matrix, we defined five dataset groups: (1) Escherichia coli (E. coli UTI89, E. coli Sakai, E. coli EDL933, E. coli ATCC 8739 and E. coli K12); (2) fruit fly (Drosophila melanogaster, D. sechellia, D. simulans, D. erecta, D. yakuba); (3) rice (Oryza indica and O. sativa); (4) mammal (human, chimp and mouse); and (5) yeast (Saccharomyces cerevisiae, S. paradoxus, S. mikatae, and S. bayanus). For the first four groups, proteins sequences and their corresponding orthologous relationships were downloaded from Ensembl Genomes (Release 4; ftp://ftp.ensemblgenomes. org) and BioMart (http://www.biomart.org), respectively. For the fifth group (yeast), proteins sequences and their corresponding orthologous relationships were retrieved from Fungal Orthogroups Repository (version 1.1; http://www.broadinstitute.org/regev/orthogroups/). Oneto-many or many-to-many orthologous relationships were excluded from this analysis, in order to avoid ambiguous or questionable orthologs. We then aligned protein sequences for each dataset group by using T-Coffee [71] and alignments with identity $<85 \%$ were removed from this analysis. As a result, we obtained orthologous alignments of 3119 in E. coli, 6302 in fruit fly, 16606 in rice, 6606 in mammal and 2026 in yeast. Finally, based on these orthologous alignments for each group, we constructed five amino acid exchange matrices.

\section{Reviewers' comments}

Reviewer's report 1

Zhaolei Zhang, Donnelly Centre for Cellular E Biomolecular Research (CCBR), University of Toronto, Toronto, Canada (nominated by Mark Gerstein, Department of 
Molecular Biophysics and Biochemistry, Program in Computational Biology and Bioinformatics, Yale University, Connecticut, USA)

\section{Major comments}

I thought the Introduction could be further clarified, please explicitly present and describe the Chargaff's first and second rules (instead of just citing the papers, ref 39. 40). And please formerly define "purine content" and explain what is the expected value for it.

Authors' response We expanded our descriptions on the Chargaffs rules and purine content in the Introduction.

I don't think it is $\mathrm{S}$ and $\mathrm{R}$ are independent, since $\mathrm{S}=$ $\mathrm{G} / \mathrm{C}$ and $\mathrm{R}=\mathrm{A} / \mathrm{G}$. It may not affect the analysis, but the authors need to clarify this.

Authors' response As defined, statistical independence of two variables means that the occurrence of one variable makes it neither more nor less probable that the other occurs. GC and purine contents are assumed independent in this study, which is based on Chargaff's rules that for simplicity, $A=T$ and $G=C$; therefore, purines are expected to be $50 \%$ since $A+T+G+C=2(A+G)=$ $100 \%$, in contrast to broad variation of GC content. That is, in theory, GC (purine) content does not influence purine (GC) content variation. In reality, it is observed that species with very close purine (or GC) contents have variations in their GC (or purine) contents. For example, Streptococcus mutans UA159 and Rubrobacter xylanophilus DSM 9941 are similar in purine content (52\%) but different in GC content ( $38 \%$ and $71 \%$, respectively), and Bartonella quintana str. Toulouse and Clostridium thermocellum ATCC 27405 are similar in GC content (40\%) but different in purine content (50\% and 57\%, respectively). Although $G C$ and purine contents share nucleotide $G$ in common, it does not mean that they are dependent. Suppose that two contents $X$ and $Y$ have no nucleotide in common, viz., mutually exclusive, for example, GC and AT, the increase (or decrease) of one content leads to the decrease (or increase) of the other. If two contents are mutually exclusive, they cannot be independent and vice versa [65]. We clarified this point more clearly in the Models.

Here the null model is that the frequency of a codon is the product of the frequency of individual nucleotide at each position. This is not very accurate since it is known genomes have severe dinucleotide biases.

Authors' response Our models adopt GC (S) and purine (R) contents to model genome compositional dynamics for nucleotides, codons and amino acids and therefore, dinucleotide bias is in fact represented primarily at the nucleotide level. Although it is not fully considered in codon composition, dinucleotide bias is accurately captured at the nucleotide level. For instance, given a sequence containing severe dinucleotide bias, CGCGCG......, it is observed that $S=100 \%$ and $R=50 \%$. According to equations 1-4, the expected contents of four nucleotides are $A=(1-S) R=0, T=(1-S)(1-R)=0, G=S R=50 \%$, and $C=S(1-R)=50 \%$, which is consistent with the observed compositions and captures well the severe dinucleotide bias.

Can the authors elaborate on these empirical relations between $\mathrm{S}$ and $\mathrm{S}_{i}$ ? Are you referring to the observed ratio between these two variables? I see the authors provided some details in the Discussion session (page 11, 12), it may help the readers to move them up front.

Authors' response Empirical relationships between $S$ and $S_{i}$ are not the observed ratio between the two variables, but the linear relationships derived from all genomes in individual domains of life (Additional file 1). We accepted the reviewer's suggestion to move the text from the Discussion to the Models and elaborated on the description of the empirical relationships more clearly.

I can understand that the GC\% could be mostly uniform along the chromosome for prokaryotes and lower eukaryotes such as fly and nematodes; but for vertebrates like frog, chicken and mammals, it is known the chromosomes can be broken into regions $(100 \mathrm{~Kb}$ at least) of uniform GC content, i.e. the so-called "isochore" concept. Also different chromosomes may have slightly different GC\%, at least in mammalian genomes. It appears that the author took the GC\% of the entire genome to predict nucleotide frequency. At least the authors should comment on this issue and how this would influence the analysis (by doing some simple experiments). Perhaps this is why the scatter plots for eukaryotes are not as linear as for bacteria and archaea.

Authors' response We agree. We added the description on the influence of isochores on eukaryotes in the Discussion and accordingly cited two relevant references (Oliver, J.L., Bernaola-Galvan, P., Carpena, P. and Roman-Roldan, $R$. 2001. Isochore chromosome maps of eukaryotic genomes. Gene. 276: 47-56.; Bernardi, G. 2000. Isochores and the evolutionary genomics of vertebrates. Gene. 241: 3-17.)

I understand and agree with the results presented, i.e. the nucleotide and codon compositions are influenced by $\mathrm{GC} \%$ and purine content (AG\%), but it is not clear to me how the authors can link GC\% with mutations, and purine content variation with selections. The authors need to provide sufficient background on this, instead of referring the readers to a list of references.

Authors' response In the context of protein-coding sequences, mutation primarily creates new substitutions, which leads to a broad variation in GC content. The same substitutions are often selected and cater for a particular amino acid composition with specific physicochemical properties or functional consequences in biologists' term. As reported by several studies and mentioned in our manuscript, purines also have a determinative role in amino acid physicochemical properties. 
Therefore, purine content should also be relevant with selection although it may be limited to a narrow range of content variation. We expanded our description on this point in the Discussion.

Minor issues not for publication

Notation: I suggest the authors use the notation of GC $\%$ instead of GC when referring to the nucleotide content.

Page 2, line 4, "have been allowing us" -> "have allowed us"

Page 2, line 6, quantization $\rightarrow$ quantification

Page 2, line 9, "take into account"

Page 6, line 15, typo "their the"

Page 8, line 11, typo "again consistent"

Authors' response We addressed the minor issues in the revised manuscript.

\section{Reviewer's report 2}

Guruprasad Ananda, Huck Institute for the Life Sciences, Pennsylvania State University, Pennsylvania, USA (nominated by Kateryna Makova, Department of Biology, Pennsylvania State University, Pennsylvania, USA)

This manuscript describes two models to quantify genome compositional dynamics for a wide range of genomes, both of which incorporate GC and purine contents as compositional parameters. These models are evaluated by comparing predicted with observed compositions across three domains of life and these comparisons yield a number of (mostly) descriptive results. First, this work demonstrates the combined role of GC content and purine content in contributing to different compositions at the levels of nucleotides, codons and amino acids. Next, it reiterates previous findings about the role of mutations and selection acting at the nucleotide-level in causing differences between predicted and observed compositions. Interestingly, the deviation of observed from expected compositions is decoupled for the three codon-positions and underlying nucleotides (A/T vs. C/G) and is used as a measure of the strength of selection acting at each position/nucleotide. These deviations are also used to assess the degree of selection acting on different amino acids, the results of which seem closely consistent with other findings.

Although the models are described quite well, the same cannot be said about results and discussion. In particular, since the deviation of observed from expected compositions is used to derive biological inferences, I think it'd be good to provide some sort of a quantitative measurement that gives an idea about the extent of this deviation. For instance, since the deviations are used to assess the degree of selection at different codon positions, having a value to these deviations can help in understanding the relative quantitative differences in the strengths of selection at the three codon-positions.

\section{Authors' response}

This study focuses on modeling genome compositional dynamics and we described the deviations of the observed from the expected compositions in the Discussion. Further investigation of these deviations is attractive, but beyond the scope of this study. This suggestion would be an excellent suggestion for next step. In fact, these deviations can be estimated by several measures (e.g., Euclidean distance, cosine similarity, Kullback-Leibler divergence) and we have been working on an algorithm to statistically quantify these deviations, which will be summarized into another paper soon.

The paper does a decent job at describing the role of varying $\mathrm{GC}$ content as a determinant of compositions at all levels - as seen in Results, Discussion and Figures. However, the same cannot be said about the role of purine content. The graphs of expected vs. observed compositions are always plotted for GC content, although the models use both GC and purine contents as parameters. One doesn't get a clear picture of the effect of purine content on compositional dynamics. This therefore clouds the results to an extent.

\section{Authors' response}

Compared to GC content, purine content varies at a relatively small range and the role of purine content in determining compositions is not significant as GC content. However, it does not mean that purine content is not important. The small range of purine content owes to strong selection for particular amino acids, since purine content has a determinative role in amino acid physic-chemical properties. Its slight departure from 50\% can lead to diversely different compositions, as indicated by equations 1-8. We clarified our description on purine content in the Discussion.

It is mentioned that Alanine and Valine are the two most departed amino acids (in the Discussion section, 6th paragraph). From Fig. S5, it becomes apparent why Valine is listed as one of the most departed amino acids; however it's hard to draw this inference for Alanine. As mentioned above, quantifying the deviations can help better understand such differences. Also, it would be interesting to provide a table indicating the amount of deviation per amino acid, and one can then easily compare this information with the data from the exchangeability matrices to understand to what extent the two are correlated.

\section{Authors' response}

We agree that providing the expected and observed frequencies of amino acids would help better understand the deviations. Thus, we summarized the information as Additional file 5.

On the whole, I would recommend this manuscript to be published as a Research article in Biology Direct with the aforementioned modifications. 
Minor issues:

Results, 1st paragraph, 6th line: of empirical...

Results, 3rd paragraph (Codon composition),

5th line: one of the codons listed doesn't match the ones in Figure 3. Replace CTC with CTT.

10th line: It should be TGC and GCC that correlate positively with GC content, right?

11th line: consistent with the observed...

Discussion, 5th paragraph (Considering nucleotide compositions.......)

Although this part of the discussion is quite important and interesting, it is not well phrased. Please try to break up the long sentence about A/C vs. G/T.

Also the terms 'across' and 'between' the positions are slightly misleading. Please rephrase.

\section{Authors' response}

We accepted the minor issues and corrected the wording accordingly.

\section{Reviewer's report 3}

Daniel Haft, The J. Craig Venter Institute, Rockville, Maryland, USA

Revised review of Daniel Haft:

Evolutionary change to genomic content includes mechanisms of lateral gene transfer, duplication, gene loss, insertion, deletion, and point mutation, all of which can alter the GC content of an organism. The most common point mutations, but by no means the only ones, are transitions, in which a nucleotide maintains its identity as a purine, or as a pyrimidine, but base pairs switch between AT and GC. Some organisms show extremely strong biases to either AT richness or GC richness, especially between genes and in the third position of each codon.

An earlier study by Knight, Freeland, and Landweber asks whether selection, with its effects on amino acid usage and their encoding by particular codons, drives GC bias in coding regions, or whether mutational biases towards a particular GC content more strongly drive patterns of codon usage and amino acid usage. The difference between these two models is that viewing GC mutational bias as the driver would allow a very simple equation to predict most of the variance seen in codon frequencies and amino acid frequencies from species to species. Knight, et al. indeed find that the single parameter of GC content can explain $71-87 \%$ of the variance across species in the differential usage of synonymous codons for a given amino acid, and $71-79 \%$ of the variance in the usage of different amino acids. The fact that codon positions 1,2 , and 3 respond so differently to overall coding region GC content in their models shows a balance between forces of mutation and selection. Their work, on the whole, nicely reveals this balance through examination of GC content alone.
The present study largely reiterates the work of Knight, Freeland, and Landweber, although coding regions for analysis are taken from roughly twice as many species, and graphs are shown for every codon and every amino acids in supplementary figures. In this study, it is noted that average purine content in coding regions, which represent just one strand at a time, may vary slightly in a narrow band around $50 \%$, with the extremes among 686 bacteria never going below $48 \%$ or above $59 \%$. The deviation of purine content from $50 \%$, therefore, theoretically allows for a slight enhancement of the power of models based only on GC content to predict actual codon frequencies and therefore amino acid frequencies. However, the authors do not quantify the degree to which adding consideration of coding strand purine content explains additional variance in codon and amino acid frequencies. Without some quantifications of how much purine content improves the models, it is impossible for readers to judge the central claims of the paper. For this reason, the work being reported here is incomplete.

\section{Authors' response}

The purpose of this paper is to model compositional dynamics. GC and purine contents are regarded as two essential parameters in quantifying compositions, e.g., for four nucleotides, $A=(1-S) R, T=(1-S)(1-R), G=S R, C=$ $S(1-R)$. It can be seen from these equations that purine content has an important contribution to the model; as mentioned in Models, "a special case when $R=0.5$, can lead to ". Our model for this special case when $R=0.5$, is equivalent to the model proposed by Knight et, al (2001) that considers GC content alone.

The paper has several serious errors in its exposition and response to review comments.

1. The computational method relies on a change of coordinates for describing base composition from one based on $\mathrm{T}, \mathrm{A}, \mathrm{G}$, and $\mathrm{C}$ to one described by just two variables, $\mathrm{GC}$ content and purine content. Changing coordinate systems is fine, but calling the two measures "independent" is not, especially because of the logic used: "According to the Chargaff's rules again, $\mathrm{S}$ varies broadly whereas $\mathrm{R}$ always centers at $50 \%$, implying the independence between $\mathrm{S}$ and R." This becomes an empty rationale for claiming independence when the actual deviation from $50 \%$ becomes a main theme of the paper. The deviation of purine content from 50\%, and the deviation of codon position $2 \mathrm{GC}$ content from overall GC content have many of the same drivers, and are not independent. It would be better simply to say that equations 1-8 allow for the four nucleotide frequencies to be described by just two parameters.

\section{Authors' response}

We agree purine content deviates from 50\%, which can be observed across the sequences. The statement "Purine 
content always centers on 50\%" is theoretically derived from Chargaff's rules that $A=T$ and $G=C$, and thus $A$ $+T+G+C=2(A+G)=100 \%$. In practice, although purine content is not always $50 \%$, the deviation range is relatively small, nearly $10 \%$ below or above $50 \%$, whereas GC content varies broadly from $\sim 20 \%$ to $\sim 80 \%$. The assumption of the independence of $G C$ and purine contents, therefore, is based on practical observations. For example, in bacteria, Streptococcus mutans UA159 and Rubrobacter xylanophilus DSM 9941 are similar in purine content (52\%) but different in GC content (38\% and $71 \%$, respectively), and Bartonella quintana str. Toulouse and Clostridium thermocellum ATCC 27405 are similar in GC content (40\%) but different in purine content (50\% and $57 \%$, respectively).

2. The response to reviewer 1 about dinucleotide bias is deeply flawed. The claim is that GCGCGC is handled correctly, but in fact it is handled the same as GGGCCC, despite the different codons and amino acid frequencies that result. Any evolutionary process that drives an alteration in dinucleotide frequencies (for a given GC content) will cause their predictive models to be off systematically. Fits by the models, in fact, are not as good as suggested qualitatively in the discussion. The codons selected for display in Figure 3 as representative are much better behaved than some seen in supplementary figure 4, where ratios of observed to predicted codon frequencies are off sometimes by two-to-one. It might be more prudent to admit that evolutionary mechanisms that introduce dinucleotide biases may be additional drivers of skewed codon frequencies, but are beyond the scope of the current work.

\section{Authors' response}

We agree that the deviations of the expected compositions from the observed result from a complex interplay of mutation and selection and relate closely to evolutionary mechanisms, such as, dinucleotide abundance. We accepted the reviewer's point to expand our discussion on these issues in the Discussion and cite a relevant reference "Karlin and Burge (1995). Trends Genet. 11: 283-290".

3. Reviewer 2 joined me in requesting quantitative measures of how well the models fit. The response that the "algorithm to statistically quantify these deviations ... will be summarized into another paper soon" is not satisfying. Modeling why the fits are wrong may be beyond the scope of this paper, but measuring whether the models fit is actually essential.

\section{Authors' response}

Sequences from different species undergo differential evolutionary processes, consequently resulting in diverse compositions, even for sequences having same GC and purine contents. For different sequences, therefore, the fitness of the models would vary at different degrees, suggesting diverse interplays of mutation and selection forces acting on these sequences. For a given composition, the models fit differently across different sequences, which can be influenced by several factors, most likely linked to the structure of the genetic code and physicochemical properties of amino acid, protein structure, the abundance of iso-accepting transfer RNAs, and translation efficiency and/or accuracy. To focus on our topics on modeling compositions, we only limited the description by taking the deviations of Ala and Val as examples in the Discussion.

4. The authors persist in describing collections of coding region sequences as "genomes" throughout much of the manuscript, when "collected coding region sequences" would be better.

\section{Authors' response}

We accepted the reviewer's suggestion and revised our wording throughout the manuscript.

Overall, this paper does not make a strong case that tracking purine content in coding regions provides useful new insights or new working models for the study of molecular evolution.

\section{Additional material}

Additional file 1: Correlations between genome-wide GC content and GC contents at three codon positions and between genomewide purine content and purine contents at three codon positions

Additional file 2: Expected and observed nucleotide compositions across the three domains of life (46 archaea, 686 bacteria, and 826 eukaryotes).

Additional file 3: Expected and observed codon compositions across the three domains of life (46 archaea, 686 bacteria, and 826 eukaryotes)

Additional file 4: Expected and observed amino acid compositions across the three domains of life (46 archaea, 686 bacteria, and 826 eukaryotes)

Additional file 5: Comparison between expected and observed amino acid compositions.

Additional file 6: Amino acid exchange matrices in Escherichia coli, fruit fly, rice, yeast, and mammal.

\section{Acknowledgements}

We thank the reviewers for their critical comments and constructive suggestions on this manuscript and Prof. Mark Gerstein and Prof. Kateryna Makova for their excellent nominations. We also thank Peng Cui, Feng Ding, Lijia Ma, Jun Li, and Ang Li for their helpful discussions and Francesc LopezGiraldez for help on data collection. This work was supported by a faculty fund from King Abdullah University of Science and Technology (KAUST) awarded to JY.

\section{Authors' contributions}

ZZ designed the two models and drafted the manuscript. JY supervised the research and revised the manuscript. Both authors read and approved the final manuscript.

\section{Competing interests}

The authors declare that they have no competing interests. 
Received: 16 July 2010 Accepted: 8 November 2010

Published: 8 November 2010

\section{References}

1. Sueoka N: On the genetic basis of variation and heterogeneity of DNA base composition. Proc Natl Acad Sci USA 1962, 48:582-592.

2. Ikemura T: Correlation between the abundance of Escherichia coli transfer RNAs and the occurrence of the respective codons in its protein genes: a proposal for a synonymous codon choice that is optimal for the E. coli translational system. J Mol Biol 1981, 151:389-409.

3. Gouy M, Gautier C: Codon usage in bacteria: correlation with gene expressivity. Nucleic Acids Res 1982, 10:7055-7074.

4. Sharp PM, Li WH: The codon Adaptation Index-a measure of directional synonymous codon usage bias, and its potential applications. Nucleic Acids Res 1987, 15:1281-1295.

5. Bennetzen JL, Hall BD: Codon selection in yeast. Journal of Biological Chemistry 1982, 257:3026-3031.

6. Ikemura T: Codon usage and tRNA content in unicellular and multicellular organisms. Mol Biol Evol 1985, 2:13-34.

7. Moriyama EN, Powell JR: Codon usage bias and tRNA abundance in Drosophila. J Mol Evol 1997, 45:514-523.

8. Akashi H: Distinguishing the effects of mutational biases and natural selection on DNA sequence variation. Genetics 1997, 147:1989-1991.

9. Vicario S, Moriyama EN, Powell JR: Codon usage in twelve species of Drosophila. BMC Evol Biol 2007, 7:226.

10. Moriyama EN, Hartl DL: Codon usage bias and base composition of nuclear genes in Drosophila. Genetics 1993, 134:847-858.

11. Murray EE, Lotzer J, Eberle M: Codon usage in plant genes. Nucleic Acids Res 1989, 17:477-498.

12. Wang $H C$, Singer GA, Hickey DA: Mutational bias affects protein evolution in flowering plants. Mol Biol Evol 2004, 21:90-96.

13. Bernardi G, Mouchiroud D, Gautier C: Compositional patterns in vertebrate genomes: conservation and change in evolution. $J \mathrm{Mol}$ Evol 1988, 28:7-18.

14. Wolfe KH, Sharp PM: Mammalian gene evolution: nucleotide sequence divergence between mouse and rat. J Mol Evol 1993, 37:441-456.

15. Bulmer M: The selection-mutation-drift theory of synonymous codon usage. Genetics 1991, 129:897-907.

16. Akashi H: Codon bias evolution in Drosophila. Population genetics of mutation-selection drift. Gene 1997, 205:269-278.

17. Chen SL, Lee W, Hottes AK, Shapiro L, McAdams HH: Codon usage between genomes is constrained by genome-wide mutational processes. Proc Natl Acad Sci USA 2004, 101:3480-3485.

18. Kanaya S, Yamada Y, Kinouchi M, Kudo Y, Ikemura T: Codon usage and tRNA genes in eukaryotes: correlation of codon usage diversity with translation efficiency and with CG-dinucleotide usage as assessed by multivariate analysis. J Mol Evol 2001, 53:290-298.

19. Akashi H: Synonymous codon usage in Drosophila melanogaster: natural selection and translational accuracy. Genetics 1994, 136:927-935.

20. Sharp PM, Emery $L R$, Zeng $K$ : Forces that influence the evolution of codon bias. Philos Trans R Soc Lond B Biol Sci 2010, 365:1203-1212.

21. Stoletzki N, Eyre-Walker A: Synonymous codon usage in Escherichia coli: selection for translational accuracy. Mol Biol Evol 2007, 24:374-381.

22. Xia X: Maximizing transcription efficiency causes codon usage bias. Genetics 1996, 144:1309-1320

23. Bernardi G: Compositional constraints and genome evolution. J Mol Evol 1986, 24:1-11.

24. Xia X: Data Analysis in Molecular Biology and Evolution Boston: Kluwer Academic Publishers; 2000.

25. Karlin S, Mrazek J: Compositional differences within and between eukaryotic genomes. Proc Natl Acad Sci USA 1997, 94:10227-10232.

26. Karlin S, Campbell AM, Mrazek J: Comparative DNA analysis across diverse genomes. Annu Rev Genet 1998, 32:185-225.

27. Nekrutenko A, Li WH: Assessment of compositional heterogeneity within and between eukaryotic genomes. Genome Res 2000, 10:1986-1995.

28. Karlin S, Mrazek J, Campbell AM: Compositional biases of bacterial genomes and evolutionary implications. J Bacteriol 1997, 179:3899-3913.

29. Zhao $X$, Zhang Z, Yan J, Yu J: GC content variability of eubacteria is governed by the pol III alpha subunit. Biochem Biophys Res Commun 2007, 356:20-25.
30. Ermolaeva MD: Synonymous codon usage in bacteria. Curr Issues Mol Biol 2001, 3:91-97.

31. Knight RD, Freeland SJ, Landweber LF: A simple model based on mutation and selection explains trends in codon and amino-acid usage and GC composition within and across genomes. Genome Biol 2001, 2: RESEARCH0010.

32. Muto A, Osawa S: The guanine and cytosine content of genomic DNA and bacterial evolution. Proc Natl Acad Sci USA 1987, 84:166-169.

33. Gentles AJ, Karlin S: Genome-scale compositional comparisons in eukaryotes. Genome Res 2001, 11:540-546.

34. Bharanidharan D, Bhargavi GR, Uthanumallian K, Gautham N: Correlations between nucleotide frequencies and amino acid composition in 115 bacterial species. Biochem Biophys Res Commun 2004, 315:1097-1103.

35. Goodarzi H, Torabi N, Najafabadi HS, Archetti M: Amino acid and codon usage profiles: adaptive changes in the frequency of amino acids and codons. Gene 2008, 407:30-41.

36. Sorimachi K, Okayasu T: Codon evolution is governed by linear formulas. Amino Acids 2008, 34:661-668

37. Hu J, Zhao X, Zhang Z, Yu J: Compositional dynamics of guanine and cytosine content in prokaryotic genomes. Res Microbiol 2007, 158:363-370

38. Sueoka N: Compositional correlation between deoxyribonucleic acid and protein. Cold Spring Harb Symp Quant Biol 1961, 26:35-43.

39. Chargaff E: Chemical specificity of nucleic acids and mechanism of their enzymatic degradation. Experientia 1950, 6:201-209.

40. Chargaff E: Some recent studies on the composition and structure of nucleic acids. J Cell Physio/ Suppl 1951, 38:41-59.

41. Foster PG, Jermiin LS, Hickey DA: Nucleotide composition bias affects amino acid content in proteins coded by animal mitochondria. $J \mathrm{Mol}$ Evol 1997, 44:282-288.

42. Bernardi G: Isochores and the evolutionary genomics of vertebrates. Gene 2000, 241:3-17.

43. Oliver UL, Bernaola-Galvan P, Carpena P, Roman-Roldan R: Isochore chromosome maps of eukaryotic genomes. Gene 2001, 276:47-56.

44. Yu J: A content-centric organization of the genetic code. Genomics Proteomics Bioinformatics 2007, 5:1-6.

45. Gu X, Li WH: A model for the correlation of mutation rate with GC content and the origin of GC-rich isochores. J Mol Evol 1994, 38:468-475.

46. Xiao JF, Yu J: A scenario on the stepwise evolution of the genetic code. Genomics Proteomics Bioinformatics 2007, 5:143-151.

47. Gu X, Hewett-Emmett D, Li WH: Directional mutational pressure affects the amino acid composition and hydrophobicity of proteins in bacteria. Genetica 1998, 102-103:383-391.

48. Foerstner KU, von Mering C, Hooper SD, Bork P: Environments shape the nucleotide composition of genomes. EMBO Rep 2005, 6:1208-1213.

49. Biro JC, Benyo B, Sansom C, Szlavecz A, Fordos G, Micsik T, Benyo Z: A common periodic table of codons and amino acids. Biochem Biophys Res Commun 2003, 306:408-415.

50. Copley SD, Smith E, Morowitz HJ: A mechanism for the association of amino acids with their codons and the origin of the genetic code. Proceedings of the National Academy of Sciences of the United States of America 2005, 102:4442-4447.

51. Lobry JR, Sueoka N: Asymmetric directional mutation pressures in bacteria. Genome Biol 2002, 3:RESEARCH0058.

52. Albu M, Min XJ, Golding GB, Hickey D: Nucleotide Substitution Bias within the Genus Drosophila Affects the Pattern of Proteome Evolution. Genome Biol Evol 2009, 2009:288-293.

53. Karlin S, Burge C: Dinucleotide relative abundance extremes: a genomic signature. Trends Genet 1995, 11:283-290.

54. Yang Z, Nielsen R: Mutation-selection models of codon substitution and their use to estimate selective strengths on codon usage. Mol Biol Evol 2008, 25:568-579.

55. Rodrigue N, Philippe $H$, Lartillot N: Mutation-selection models of coding sequence evolution with site-heterogeneous amino acid fitness profiles. Proc Natl Acad Sci USA 2010, 107:4629-4634.

56. Sueoka N: Directional mutation pressure and neutral molecular evolution. Proc Natl Acad Sci USA 1988, 85:2653-2657.

57. Hershberg R, Petrov DA: Selection on codon bias. Annu Rev Genet 2008 , 42:287-299.

58. Singer GA, Hickey DA: Nucleotide bias causes a genomewide bias in the amino acid composition of proteins. Mol Biol Evol 2000, 17:1581-1588. 
59. Seligmann H: Cost-minimization of amino acid usage. J Mol Evol 2003, 56:151-161.

60. Archetti M: Selection on codon usage for error minimization at the protein level. J Mol Evol 2004, 59:400-415.

61. Zhu $C T$, Zeng $X B$, Huang WD: Codon usage decreases the error minimization within the genetic code. J Mol Evol 2003, 57:533-537.

62. Archetti $\mathrm{M}$ : Codon usage bias and mutation constraints reduce the level of error minimization of the genetic code. J Mol Evol 2004, 59:258-266.

63. Bag SK, Paul S, Ghosh S, Dutta C: Reverse polarization in amino acid and nucleotide substitution patterns between human-mouse orthologs of two compositional extrema. DNA Res 2007, 14:141-154.

64. Tang $\mathrm{H}$, Wyckoff $\mathrm{GJ}, \mathrm{Lu} J, \mathrm{Wu} \mathrm{Cl}$ : A universal evolutionary index for amino acid changes. Mol Biol Evol 2004, 21:1548-1556.

65. Bhat BR: Independence. Modern Probability Theory. Third edition. New Delhi: New Age International; 1999, 213-230.

66. Bofkin L, Goldman N: Variation in evolutionary processes at different codon positions. Mol Biol Evol 2007, 24:513-521.

67. D'Onofrio G, Bernardi G: A universal compositional correlation among codon positions. Gene 1992, 110:81-88.

68. Majumdar S, Gupta SK, Sundararajan VS, Ghosh TC: Compositional correlation studies among the three different codon positions in 12 bacterial genomes. Biochem Biophys Res Commun 1999, 266:66-71.

69. Anisimova M, Kosiol C: Investigating protein-coding sequence evolution with probabilistic codon substitution models. Mol Biol Evol 2009, 26:255-271.

70. Nakamura Y, Gojobori T, Ikemura T: Codon usage tabulated from international DNA sequence databases: status for the year 2000. Nucleic Acids Res 2000, 28:292.

71. Notredame C, Higgins DG, Heringa J: T-Coffee: A novel method for fast and accurate multiple sequence alignment. J Mol Biol 2000, 302:205-217.

doi:10.1186/1745-6150-5-63

Cite this article as: Zhang and Yu: Modeling compositional dynamics based on GC and purine contents of protein-coding sequences. Biology Direct 2010 5:63.

\section{Submit your next manuscript to BioMed Central and take full advantage of:}

- Convenient online submission

- Thorough peer review

- No space constraints or color figure charges

- Immediate publication on acceptance

- Inclusion in PubMed, CAS, Scopus and Google Scholar

- Research which is freely available for redistribution

Submit your manuscript at www.biomedcentral.com/submit
Biomed Central 